\title{
Characterizing an Ionic Liquid as a Biological Fixative in Fluorescence Microscopy
}

\author{
Le A. Trinh ${ }^{1}$, Francesco Cutrale ${ }^{1}$, Scott E. Fraser ${ }^{1}$, Jim P. Kilcrease ${ }^{2}$ and Eduardo Rosa-Molinar ${ }^{3}$ \\ 1. University of Southern California, Molecular and Computational Biology, Translational Imaging \\ Center, Los Angeles, CA \\ 2. Hitachi High Technologies America, Incorporated, Nanotechnology Systems Division, Clarksburg, MD \\ 3. University of Kansas, Microscopy and Analytical Imaging Resource Core Laboratory, Lawrence, KS
}

In recent years, light microscopy technology has pushed the envelope to achieve higher temporal, spatial, and spectral resolution ${ }^{1-3}$. These advance techniques have enabled fluorescence microscopy to provide qualitative and quantitative readouts of cellular and molecular phenotypes in fixed and live samples and has transformed cell and molecular data ${ }^{4,5}$. However, efforts to achieve higher resolution in optical microscopy and realize its full potential are hampered by the obstacle presented by the diffraction limit of light. To overcome this challenge, Correlative Light and Electron Microscopy (CLEM) is being used to combine fluorescence and electron microscopy (EM).

CLEM implementation requires sample preparation protocols that allow seamless transition between light and electron imaging modalities. However, many fixatives used for EM are not compatible with fluorescence microscopy as they can quench fluorescence signal or introduce new fluorescence spectra to the biological sample. Additionally, fixation of tissue can distort cell morphology and affect protein localization $^{6}$, leading to distortion of subcellular structures. To identify fixatives that are more compatible for CLEM, we explored the use of an ionic liquid, IL1000 ${ }^{7}$, for fluorescence microscopy.

To test the effects of IL1000 on biological samples in fluorescence imaging, we coupled hyperspectral imaging and phasor analysis with the imaging of transgenic zebrafish larvae that express fluorescent fusion proteins localized to subcellular compartments such as cell membrane, nuclei, and synapses (Fig. 1). Hyperspectral imaging allows the identification of multiple fluorescence spectra in a sample by capturing the full spectrum of wavelengths emitted or reflected at each pixel within the imaged data (Fig. 1A). Segmenting the pixel data by Fourier transform using the Spectral Phasor (SP) method ${ }^{8-10}$ offers an efficient representation of the hyperspectral data by condensing each spectrum in $\mathrm{x}, \mathrm{y}, \mathrm{z}$ into a single point in a 2D Phasor plot without data $\operatorname{loss}^{8}$ (Fig. 1B). Imaging live, IL1000 or formaldehyde fixed zebrafish larvae using hyperspectral mode and analyzing the datasets with a newly developed multi-platform software package, Hyper-Spectral Phasors (HySP) ${ }^{10}$, allows the identification of spectra gain or loss in samples treated with the different fixatives.

Through hyperspectral imaging and phasor analysis, we demonstrate the effects of fixatives on fluorescence spectra emitted from zebrafish larvae in fluorescence microscopy. We show that formaldehyde fixation results in autofluorescence that dominates in spectral imaging and is reflected in the Phasor plots (Fig. 2). The accentuated autofluorescence signature detected in formaldehyde treated samples was not observed in IL1000 treated samples (Fig. 2A). More importantly, IL1000 treated samples showed similar Phasor plots as live larvae, indicating the absence of spectra gain or loss as a result of IL1000 treatment (Fig. 2B). However, decreases in fluorescence intensity and changes to cell morphology were observed with the higher concentration of IL1000 treatment, suggesting the need to optimize fixative time and concentrations.

Our hyperspectral imaging and phasor analysis comparing live and fixed zebrafish larvae suggests that IL1000 provides minimal alteration to biological samples in fixation protocols for fluorescence microscopy. Although IL1000 preserves fluorescence spectra in treated samples, the observed morphological changes 
and decrease in fluorescence signal (Fig. 2B) suggest the need to improve IL1000 for use in embryonic tissue.

\section{References:}

[1] Kanchanawong, P. \& Waterman, C.M. Curr Opin Cell Biol 24, (2012), 125

[2] Rust, M.J., Bates, M. \& Zhuang, X. Nat Methods 3, (2006), 793.

[3] Sengupta, P., van Engelenburg, S.B. \& Lippincott-Schwartz, J. Chem Rev 114, (2014), 3189.

[4] Lippincott-Schwartz, J. Mol Biol Cell 25, (2014), 3437.

[5] Muzzey, D. \& van Oudenaarden, A. Annu Rev Cell Dev Biol 25, (2009), 301.

[6] Schnell, U., et al, B.N. Nat Methods 9, (2012), 152.

[7] Kawai, K., Kaneko, K. \& Yonezawa, T. Langmuir 27, (2011), 7353.

[8] Cutrale, F., Salih, A. \& Gratton, E. Methods Appl Fluoresc 1, (2013), 35001.

[9] Favreau, P.F. et al. J Biomed Opt 19, (2014), 046010.

[10] Cutrale, F. et al. Nat Methods 14, (2017), 149.

A

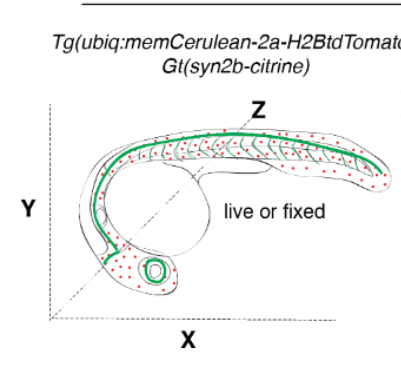

B

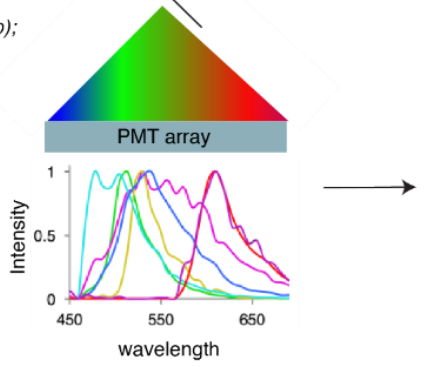

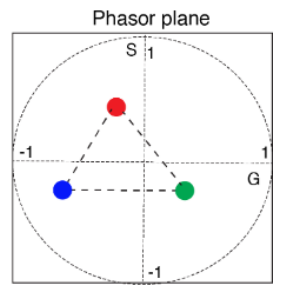

Figure 1. Hyperspectral imaging and phasor analysis. (A) Double transgenic zebrafish larvae are imaged live or fixed with different fixatives in hyperspectral mode in which a complete spectrum in the visible range is collected for each pixel $(\mathrm{x}, \mathrm{y}, \mathrm{z})$ on a photomultiplier array. (B) The multiple-dimensional $(\mathrm{x}, \mathrm{y}, \mathrm{z}, \lambda)$ dataset is Fourier transformed and represented on the phasor plot as linear combinations of $\mathrm{G}$ and $\mathrm{S}$ coordinates.

live

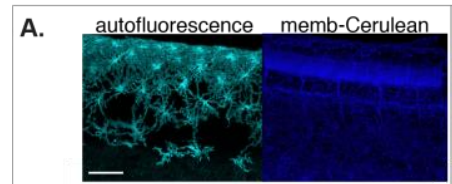

B.

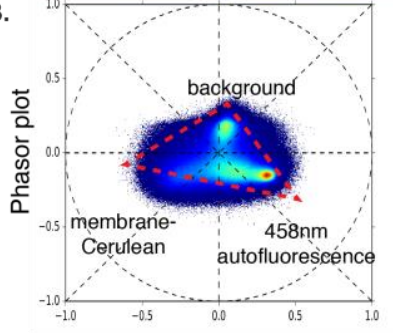

formaldehyde
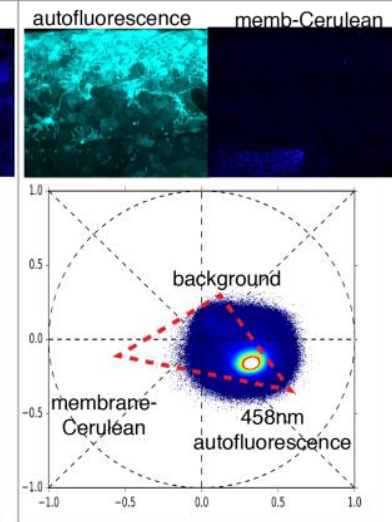

IL1000

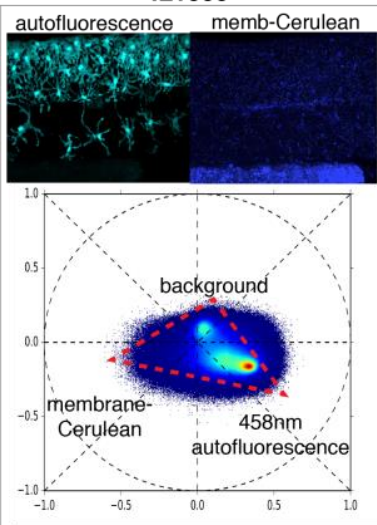

Figure 2. Effects of ionic liquid fixation on fluorescence signal in zebrafish larvae. (A) Maximum intensity projections of confocal Z-stack of live, formaldehyde or IL1000 fixed double transgenic, Tg(ubiq:memCerulean2a-H2BtdTomato;Gt(syn2b-citrine), 2dpf zebrafish larvae imaged at the trunk region with 458nm excitation and processed by Phasor to segment individual fluorescence signatures. Two of the fluorescence signatures detected in

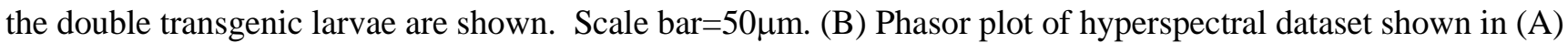
with three independent vector components highlighted by red triangle. The same three vector components in similar ratios, as represented by heat map, are seen in IL1000 treated samples as in the live larvae. However, the vector component corresponding to the membrane-cerulean signature is absent in formaldehyde treated samples, while the $458 \mathrm{~nm}$ autofluorescence dominates. 